\title{
Performance of Multi-user MIMO Precoding with Limited Feedback over Measured Channels
}

\author{
Florian Kaltenberger, David Gesbert, Raymond Knopp \\ Eurecom \\ 2229, Route des Cretes - B.P. 193 \\ 06904 Sophia Antipolis, France
}

\author{
Marios Kountouris \\ Wireless Networking and Communications Group \\ The University of Texas at Austin \\ Austin, TX 78712, USA
}

\begin{abstract}
In multi-user multiple-input multiple-output (MUMIMO) systems, channel state information at the transmitter (CSIT) allows for multi-user spatial multiplexing and thus increases the system throughput. We assume that CSIT is obtained by means of a finite-rate feedback channel through channel vector quantization (CVQ) at the receiver. In this paper we use real channel measurements to study the effect of CVQ on the sum rate of a MU-MIMO system employing linear precoding. The measurement data has been acquired using Eurecom's MIMO Openair Sounder (EMOS). The EMOS can perform realtime MIMO channel measurements synchronously over multiple users. We consider CVQ using a Fourier codebook, a random codebook and a random codebook exploiting the second order statistics of the channel. For comparison, we also show the capacity of a single-user system using time division multiple access (TDMA) with no CSIT at all. The results show that the Fourier codebook shows very poor performance in the measured channels. Random codebooks - although suboptimal-provide a much better performance in the measured channels.
\end{abstract}

\section{INTRODUCTION}

We study the downlink (broadcast) channel of a wideband multi-user multiple-input multiple-output (MU-MIMO) system with multiple antennas at the base-station (BS) and possibly multiple antennas at the user equipment (UE). Information theory reveals that if there is full channel state information at the transmitter (CSIT) and the receiver (CSIR), the optimum transmit strategy for the MU-MIMO broadcast channel involves a theoretical pre-interference cancellation technique known as dirty paper coding (DPC) combined with an implicit user scheduling and power loading algorithm [1], [2].

While the assumption of good CSIR can be justified by the ability of the receiver to estimate the channel using training symbols on the downlink, perfect CSIT is almost impossible to achieve. However, it is possible to obtain partial CSIT by means of a limited feedback channel as envisioned in, for example, 3GPP long term evolution (LTE). For a state of the art on this topic, we refer the interested reader to [3], [4].

There are many possible choices of what information to feed back and an optimal solution has yet to be found. Also, given a certain feedback strategy, it is not trivial how to use this feedback at the transmitter in an optimal way. One solution that has also been proposed for 3GPP LTE is channel

This research was supported by the project PACAM with SFR, the EC under FP7 Network of Excellence project NEWCOM++ and Eurecom. vector quantization (CVQ) along with linear precoding at the transmitter [5], [6].

In this paper we use real channel measurements to study the effect of CVQ on the sum rate of such a MU-MIMO system. We consider CVQ using a Fourier codebook, a random codebook and a random codebook exploiting the second order statistics of the channel. We compute the achievable sumrate of these schemes using a standard linear MU-MIMO precoder such as zero-forcing $(\mathrm{ZF})$ and regularized inversion (also called MMSE precoder) [7]. For comparison we also study the performance of MU-MIMO linear precoding based on full CSIT and a single-user multiple-input single-output (MISO) time division multiple access (TDMA) system with full CSIT as well as no CSIT.

MU-MIMO channel measurements have been obtained using Eurecom's MIMO Openair Sounder (EMOS) [8]. The EMOS can perform real-time channel measurements synchronously over multiple users moving at vehicular speed. The measured channels are stored to disk for offline analysis. For this paper, we have used four transmit antennas and four users with one antennas each. The channel measurements have been used in [9] to evaluate the capacity of linear multi-user MIMO precoding schemes assuming a perfect feedback channel. The spatial correlation of the measured channels has been studied in [10] and the results will be used in this paper to generate the random codebooks.

To the best of our knowledge, no such comparison based on real MU channel measurements has been reported. Real indoor channel measurements have been used in [11] for the evaluation of the proposed MU-MIMO scheme. Real outdoor channel measurements have been used in [12] to study limited feedback. However, the channel measurements were obtained with one receiver at different times and not synchronously as in our measurements.

Paper Organization: We introduce the signal model in Section II. The investigated channel vector quantization and MU-MIMO linear precoding schemes are given in III. In Section IV we describe the EMOS in some more detail and explain how the channel measurements are performed. In Section V the measurement campaign is described and results are discussed. We finally give conclusions in Section VI.

Notation: Column vectors and matrices are denoted by a and $\mathbf{A}$ respectively. $\mathbf{I}_{M}$ is the identity matrix of size $M$ and 
$\mathbf{0}_{M}$ is an $M$-dimensional vector of zeros. The Euclidean $\left(\ell_{2}\right)$ norm of a vector a is denoted by $\|\mathbf{a}\| . \mathbb{E}$ denotes expectation, and $\mathcal{C N}(\mathbf{m}, \mathbf{C})$ denotes a multivariate proper complex normal distribution with mean vector $\mathbf{m}$ and covariance matrix $\mathbf{C}$.

\section{SySTEM MOdeL}

We consider a multi-user, multi-antenna downlink channel in which a BS equipped with $M$ antennas communicates with $K \leq M$ terminals, each equipped with one antenna. The received signal $y_{k, m, q} \in \mathbb{C}$ of the $k$-th user at time $m$ and frequency $q$ is mathematically described as

$$
y_{k, m, q}=\mathbf{h}_{k, m, q}^{T} \mathbf{x}_{m, q}+n_{k, m, q} \quad \text { for } \quad k=1, \ldots, K
$$

where $\mathbf{h}_{k, m, q} \in \mathbb{C}^{M}$ represents the $k$-th user channel response at time $m$ and frequency $q, \mathbf{x}_{m, q} \in \mathbb{C}^{M}$ is the vector of transmitted symbols at time $m$ and frequency $q$, and $n_{k, m, q} \in \mathbb{C}$ is i.i.d. circularly symmetric additive complex Gaussian noise with zero mean and variance $\sigma^{2}, \forall k$.

Equation (1) can also be written in matrix notation by defining $\mathbf{H}_{m, q}=\left[\mathbf{h}_{1, m, q} \ldots \mathbf{h}_{K, m, q}\right]^{T}$ and the vectors $\mathbf{y}_{m, q}$ and $\mathbf{n}_{m, q}$ accordingly:

$$
\mathbf{y}_{m, q}=\mathbf{H}_{m, q} \mathbf{x}_{m, q}+\mathbf{n}_{m, q} .
$$

We assume that each of the receivers has perfect and instantaneous knowledge of its own channel. Further we assume a zero-delay error-free finite-rate feedback channel with a resolution of $B$ bits for each subcarrier $q$ and time $m$. Note, that we do not exploit time or frequency correlation in the feedback encoder. The transmitter is subject to an average power constraint, i. e., $\mathbb{E}\left\{\mathbf{x}_{m, q}^{H} \mathbf{x}_{m, q}\right\} \leq P$, which implies that the total transmit power is not dependent on the number of transmit antennas. For notation convenience, in the following sections we drop the time and frequency indices.

\section{Linear Precoding With Limited Feedback}

In this section we present a MU-MIMO scheme that uses linear precoding based on quantized channel feedback. This scheme has also been proposed for UMTS-LTE [5]. Although nonlinear precoding schemes can achieve a better sum rate than linear precoding, they often exhibit more complexity and a lack of robustness with respect to imperfect CSIT. Also, it has been shown in [13], that under certain conditions linear beamforming along the quantized channel directions is optimal. For comparison we also present the capacity of a SUMISO TDMA system with and without CSIT in this section.

\section{A. Channel Vector Quantization}

For each subcarrier, the UE $k$ selects a quantization vector $\hat{\mathbf{h}}_{k}$ from a codebook $\mathcal{C}=\left\{\mathbf{c}_{1}, \ldots, \mathbf{c}_{C}\right\}$ of size $C=2^{B}$, such that the angle between the actual channel $\mathbf{h}_{k}$ and the quantized channel $\hat{\mathbf{h}}_{k}$ is minimized. In other words,

$$
\hat{\mathbf{h}}_{k}=\mathbf{c}_{n}, \quad n=\underset{i=1, \ldots, C}{\operatorname{argmax}}\left|\mathbf{c}_{i}^{H} \mathbf{h}_{k}\right| .
$$

For every subcarrier, the UE then feeds back the index $n$ along with a channel quality information (CQI). In this paper we use the channel vector norm $\left\|\mathbf{h}_{k}\right\|$ as CQI. Note that this choice of CQI is not suitable for multi-user scheduling, since it does not take the multi-user interference and the quantization error into account. However, in this paper we are only interested in the precoder design and do not consider scheduling. Moreover, we assume that the channel vector norm is not quantized, since we are only interested in the ability of the codebook to capture the spatial properties of the channel. The codebook $\mathcal{C}$ is designed off-line and there are several possibilities. In this paper we consider the following three designs: a Fourier codebook, a random codebook and a correlated random codebook.

1) Fourier Codebook: The Fourier codebook is obtained by defining $\mathbf{c}_{i}$ as the top $M$ rows of the $i$-th column of the DFT matrix of size $C$, i.e.,

$$
\mathbf{c}_{i}=\frac{1}{\sqrt{M}}\left[1, e^{-2 \pi j i / C}, \ldots, e^{-2 \pi j i(M-1) / C}\right]^{T} .
$$

The Fourier codebook lookup (3) can be implemented efficiently (in terms of memory and computation) by means of an inverse fast Fourier transform (IFFT). Also, the codebook does not need to be stored at the transmitter, as it can be easily reconstructed.

2) Random Codebook: The quantization vectors of the random codebook are drawn from an i.i.d. complex Gaussian distribution on the $M$-dimensional unit sphere, i.e., $\mathbf{c}_{i} \in$ $\mathcal{C N}\left(\mathbf{0}_{M}, \mathbf{I}_{M}\right)$ and $\left\|\mathbf{c}_{i}\right\|=1$.

3) Correlated Random Codebook: The quantization vectors of the correlated random codebook are drawn from complex Gaussian distribution on the $M$-dimensional unit sphere, whose covariance matrix matches the transmit correlation matrix of the channel, i.e., $\mathbf{c}_{i} \in \mathcal{C N}\left(\mathbf{0}_{M}, \mathbf{R}_{\mathrm{Tx}}\right)$ and $\left\|\mathbf{c}_{i}\right\|=1$. The transmit correlation matrix is defined as

$$
\mathbf{R}_{\mathrm{Tx}}=\mathbb{E}\left\{\mathbf{H}^{H} \mathbf{H}\right\} .
$$

In this paper we estimate $\mathbf{R}_{\mathrm{Tx}}$ from the measurements by taking the mean of $\mathbf{H}^{H} \mathbf{H}$ over all frequencies $q$ and all frames $m$ in one measurement [10].

Except for the Fourier codebook, we assume that each users has a different and independently generated quantization codebook as in [14].

\section{B. Linear Precoding}

Let $s_{k} \in \mathbb{C}$ denote the $k$-th user transmit symbol. Under linear precoding, the transmitter multiplies the data symbol for each user $k$ by a precoding vector $\mathbf{w}_{k} \in \mathbb{C}^{M}$ so that the transmitted signal is a linear function $\mathbf{x}=\sum_{k=1}^{K} \mathbf{w}_{k} s_{k}$. The resulting received signal vector for user $k$ is given by

$$
y_{k}=\mathbf{h}_{k}^{T} \mathbf{w}_{k} s_{k}+\sum_{j \neq k} \mathbf{h}_{k}^{T} \mathbf{w}_{j} s_{j}+n_{k},
$$

where the second-term in (6) represents the multi-user interference.

In this paper we use regularized channel inversion with equal power allocation based on the quantized channel matrix $\hat{\mathbf{H}}=\left[\hat{\mathbf{h}}_{1}, \ldots, \hat{\mathbf{h}}_{K}\right]$ to design the precoder $\mathbf{W}=\left[\mathbf{w}_{1}, \ldots, \mathbf{w}_{K}\right]$ [7]:

$$
\mathbf{W}=\hat{\mathbf{H}}^{H}\left(\hat{\mathbf{H}} \hat{\mathbf{H}}^{H}+\beta \mathbf{I}\right)^{-1},
$$




\begin{tabular}{|c|c|}
\hline Parameter & Value \\
\hline Center Frequency & $1917.6 \mathrm{MHz}$ \\
Bandwidth & $4.8 \mathrm{MHz}$ \\
BS Transmit Power & $30 \mathrm{dBm}$ \\
Number of Antennas at BS & $4(2$ cross polarized) \\
Number of UE & 4 \\
Number of Antennas at UE & 1 (out of 2$)$ \\
Number of Subcarriers & 160 \\
\hline
\end{tabular}

TABLE I

EMOS PARAMETERS

where $\beta$ is a regularization factor. The above scheme is often referred to as Minimum Mean Square-Error (MMSE) precoding with equal power allocation due to the analogous with MMSE beamforming weight design criterion if the noise is spatially white. The achievable sum rate is given by applying the precoder to the real channel $\mathbf{H}$

$$
\mathcal{R}_{\mathrm{MMSE}}(\mathbf{H})=\sum_{k=1}^{K} \log _{2}\left(1+\frac{\left|\mathbf{h}_{k} \mathbf{w}_{k}\right|^{2}}{\sum_{j \neq k}\left|\mathbf{h}_{k} \mathbf{w}_{j}\right|^{2}+K \sigma^{2} / P}\right) .
$$

where $\mathbf{w}_{k}$ is the normalized $k$-th column of the precoder given in (7).

If $\beta=0$, Equation (7) reduces to the zero forcing ( $\mathrm{ZF}$ ) precoder. However, when the channel is ill-conditioned, at least one of the singular values of $\left(\mathbf{H H}^{H}\right)^{-1}$ is very large, resulting in a very low $\mathrm{SNR}$ at the receivers. Also the $\mathrm{ZF}$ precoder is not very robust to channel estimation quantization errors [15].

A non-zero $\beta$ value on the other hand allows for a certain amount of multi-user interference. The amount of interference is determined by $\beta>0$ and an optimal tradeoff between the condition of the channel matrix inverse and the amount of crosstalk ought to be found. In practice, the regularization factor is commonly chosen as $\beta=M \sigma^{2} / P$ motivated by the results in [7] that show that it approximately maximizes the SINR at each receiver, and leads to linear capacity growth with $M$. The performance of MMSE is certainly significantly better at low SNR and converges to that of ZF precoding at high SNR. However, MMSE does not provide parallel and orthogonal channels and thus power allocation techniques cannot be performed in a straightforward manner.

\section{Time Division Multiple Access}

For completeness we will also compare the MU-MIMO sum rate (8) to a SU-MISO system, where the users are served in a time division multiple access (TDMA) fashion. We study both a scenario with perfect CSIT and no CSIT.

The capacity of a single user $k$ with full CSIT is given by

$$
\mathcal{C}_{\text {SU-CSIT }}\left(\mathbf{h}_{k}\right)=\log _{2}\left(1+\frac{P}{\sigma^{2}}\left\|\mathbf{h}_{k}\right\|^{2}\right) .
$$

The capacity is achieved by dominant eigenmode transmission [16]. The capacity of a single user $k$ with no CSIT is given by

$$
\mathcal{C}_{\text {SU-noCSIT }}\left(\mathbf{h}_{k}\right)=\log _{2}\left(1+\frac{P}{\sigma^{2} M}\left\|\mathbf{h}_{k}\right\|^{2}\right) .
$$

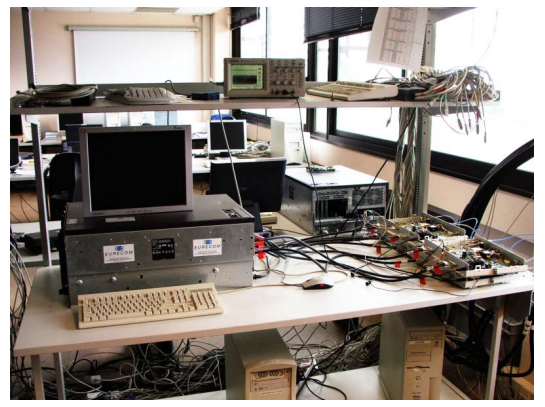

(a) Server PC with PLATON boards

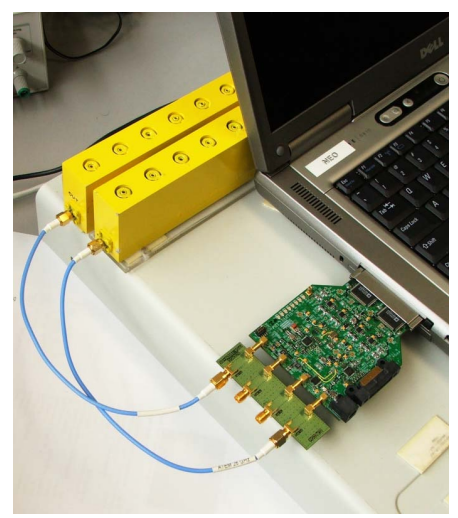

(c) Dual-RF CardBus/PCMCIA Card

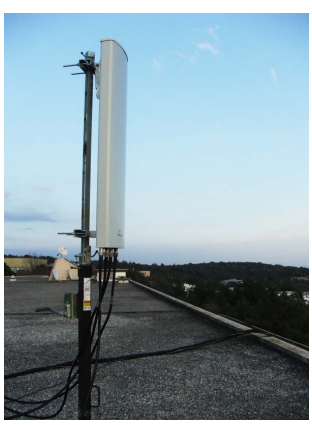

(b) Powerwave Antenna

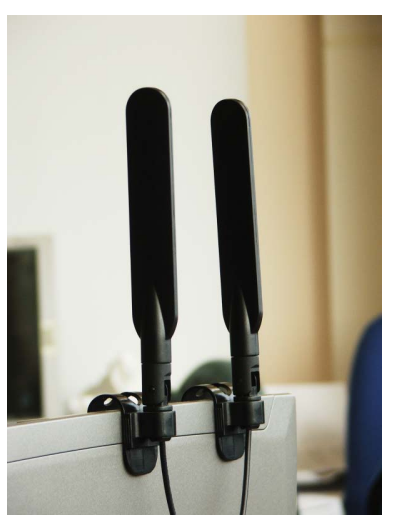

(d) Panorama Antennas
Fig. 1. EMOS base-station and user equipment [8]

The maximum sum rate capacity is achieved by transmitting to the user with the largest single-user capacity. However, in this paper we assume that all users are served fairly proportional in a round robin fashion, i. e., we treat each $\mathbf{h}_{k}$ as a different realization.

\section{The EMOS Multi-User Platform}

\section{A. Hardware Description}

The Eurecom MIMO Openair Sounder (EMOS) is based on the OpenAirInterface hardware/software development platform at Eurecom. The platform consists of a BS and one or more UEs. For the BS, a workstation with four PLATON data acquisition cards (see Fig. 1(a)) is employed along with a Powerwave $3 \mathrm{G}$ broadband antenna (part no. 7760.00) composed of four elements which are arranged in two cross-polarized pairs (see Fig. 1(b)). The UEs consist of a laptop computer with Eurecom's dual-RF CardBus/PCMCIA data acquisition card (see Fig. 1(c)) and two clip-on 3G Panorama Antennas (part no. TCLIP-DE3G, see Fig. 1(d)). The platform is designed for a full software-radio implementation, in the sense that all protocol layers run on the host PCs under the control of a Linux real time operation system.

\section{B. Sounding Signal}

The EMOS is using an OFDM modulated sounding sequence. The duration of one transmit frame is $2.667 \mathrm{~ms}$ and it consists of a synchronization symbol $(\mathrm{SCH})$, a broadcast data channel (BCH) comprising 7 OFDM symbols, a guard interval, 


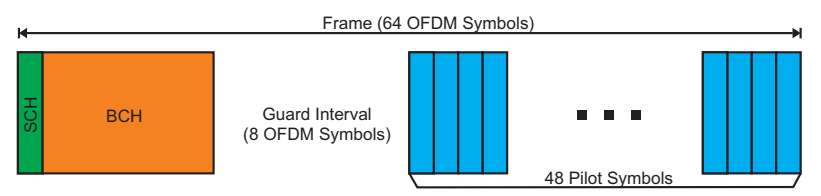

Fig. 2. Frame structure of the OFDM Sounding Sequence.

and 48 pilot symbols used for channel estimation (see Fig. 2). The pilot symbols are taken from a pseudo-random QPSK sequence defined in the frequency domain. The subcarriers of the pilot symbols are multiplexed over the four transmit antennas to ensure orthogonality in the spatial domain. The $\mathrm{BCH}$ contains the frame number of the transmitted frame that is used for synchronization among the UEs.

\section{Channel Estimation Procedure}

Each UE first synchronizes to the BS using the SCH. It then tries to decode the data in the $\mathrm{BCH}$. If the $\mathrm{BCH}$ can be decoded successfully, the channel estimation procedure is started. The channel estimation procedure consists of two steps. Firstly, the pilot symbols are derotated with respect to the first pilot symbol to reduce the phase-shift noise generated by the dualRF CardBus/PCMCIA card. Secondly, the pilot symbols are averaged to increase the measurement SNR. The estimated MIMO channel is finally stored to disk. For a more detailed description of the channel estimation see [8].

\section{Multi-user Measurement Procedure}

In order to conduct multi-user measurements, all the UEs need to be frame-synchronized to the BS. This is achieved by storing the frame number encoded in the $\mathrm{BCH}$ along with the measured channel at the UEs. This way, the measured channels can be aligned for later evaluations. The frame number is also used to synchronize the data acquisition between UEs.

\section{Measurements and Results}

\section{A. Measurement Description}

The measurements were conducted outdoors in the vicinity of the Eurecom institute. The scenario is characterized by a semi-urban hilly terrain, composed by short buildings and vegetation with a predominantly present LOS. Fig. 3 shows a map of the environment. The BS is located at the roof of Eurecom's southmost building. The antenna is directed towards Garbejaire, a small nearby village. The UEs were placed inside standard passenger cars which were being driven along the routes shown in Fig. 3. For all evaluations in this paper, we use only the first antenna at the UEs. Further, to ensure a constant average SNR of $10 \mathrm{~dB}$ at the UEs, the channel of every user is normalized over the whole measurement run (about $50 \mathrm{sec}$ ).

\section{B. Results}

Firstly, we compare the performance of MU-MIMO with MMSE precoding and CVQ to the the same scheme with perfect feedback (full CSIT). Our comparisons are based on the empirical cumulative density function (CDF) of the sum rate (cf. (Equation (8)). We show results for CVQ using

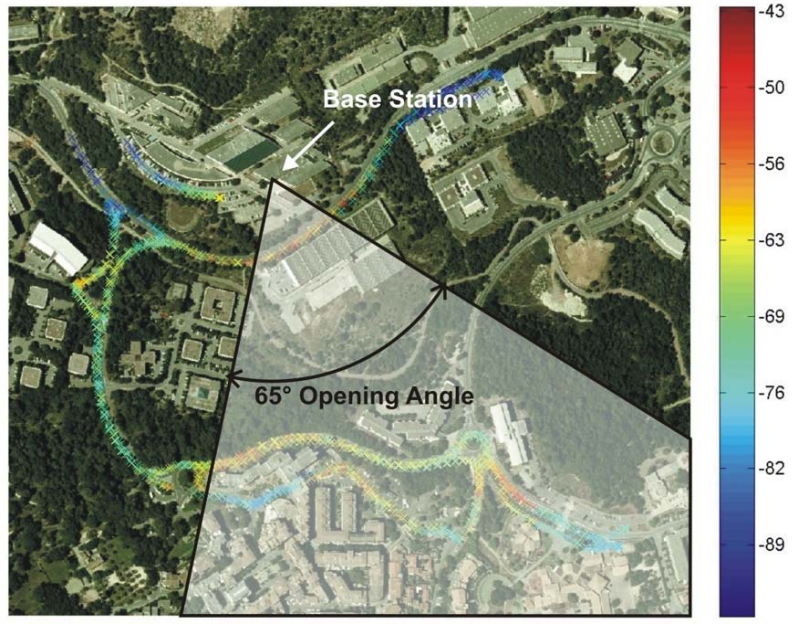

Fig. 3. Map of the measurement scenario. The position and the opening angle of the BS antenna are also indicated. The users were driving in cars along the indicated routes (the colors show the received signal strength in $\mathrm{dBm}$ along the routes).

a Fourier codebook, a random codebook, and a correlated random codebook. All schemes use 12 bits of feedback per subcarrier. The results are plotted in Figure 4 (a).

Secondly, we compare the performance of MU-MIMO with MMSE precoding and CVQ to a SU-MISO TDMA scheme with perfect feedback (full CSIT) as well as no feedback at all (no CSIT). For the latter we use the CDF of the capacity given in Equations (9) and (10). The results are plotted in Figure 4 (b).

Last but not least we compare the ergodic capacity of MUMIMO using MMSE precoding and CVQ based on different codebooks vs. the codebook size. The results are plotted in Figure 5 for various quantize resolutions.

\section{Discussion}

It can be seen that the performance of MU-MIMO with MMSE precoding depends strongly on the chosen codebook. For the evaluated outdoor channel, the Fourier codebook has the worst performance, being only slightly better than a SUMISO TDMA scheme with no feedback at all. Further, its performance does not increase with the number of feedback bits. The random codebook on the other hand performs better than the Fourier codebook for 12 feedback bits, but worse than the Fourier codebook for 4 feedback bits. The correlated random codebook performs slightly better than the one neglecting the correlation.

The performance of the correlated random codebook with 12 bits feedback is significantly higher $(2.7 \mathrm{bits} / \mathrm{sec} / \mathrm{Hz}$ at $50 \%$ outage rate) than a system with no feedback at all and even slighlty better that a SU-MISO TDMA system with perfect feedback. The gap to a MU-MIMO system with perfect feedback is approximately $1.2 \mathrm{bits} / \mathrm{sec} / \mathrm{Hz}$ (at 50\% outage rate), which is comparable to the theoretical results achieved in [14].

One explanation for the fact that the performance of the 

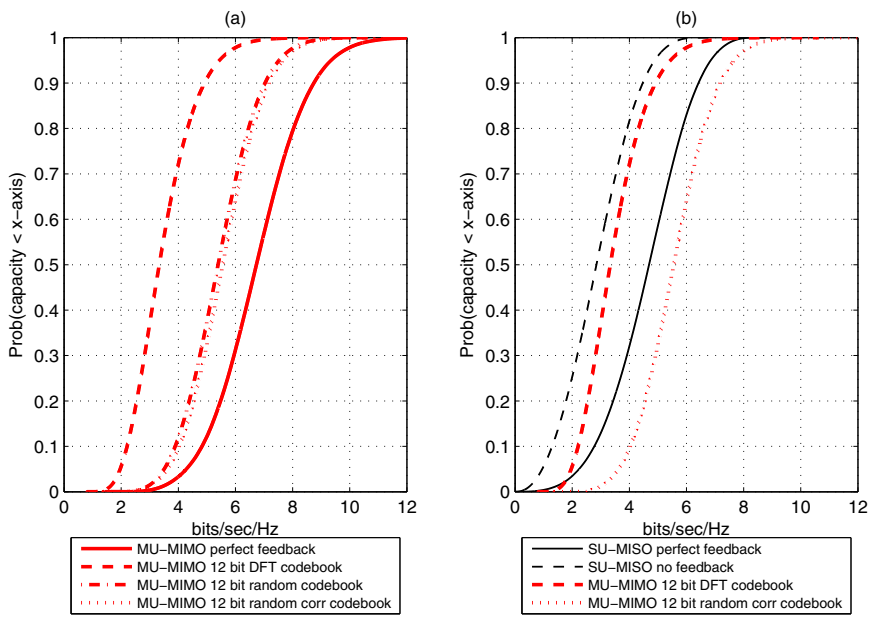

Fig. 4. CDF of the sum rate: (a) Comparing MU-MIMO with MMSE precoding and three different codebooks as well as perfect feedback, (b) Comparing MU-MIMO with MMSE precoding and two selected codebooks to SU-MISO TDMA with perfect feedback and not feedback. The average SNR is fixed to $10 \mathrm{~dB}$ for each user.

Fourier codebook does not increase with the codebook size can be obtained by looking at the maximum cross-correlation between codebook entries, $f(C)=\max _{\mathbf{c}_{i}, \mathbf{c}_{j} \in C, i \neq j}\left|\mathbf{c}_{i}^{H} \mathbf{c}_{j}\right|$. In the case of a Fourier codebook, $f(C)$ will converge to one as the codebook size increases (just choose two neighboring codebook entries $\mathbf{c}_{i}$ and $\mathbf{c}_{i+1}$ ). For the random codebook on the other hand $f(C)$ will converge to zero, since any two codewords are uncorrelated with probability one.

\section{CONCLUSiOnS}

We have presented an analysis of measured wideband MUMIMO channels with respect to the performance of linear precoding with quantized channel state information. The measurement data was acquired using Eurecom's MU-MIMO channel sounder EMOS. We have shown results for channel vector quantization employing a Fourier codebook, a random codebook and a correlated random codebook.

It can be concluded that the Fourier codebook is not able to capture the spatial properties of the measured outdoor wideband (mostly LOS) channel appropriately, since its performance does not increase with the number of feedback bits. Even for a high number of feedback bits, its performance is comparable to a SU-MISO TDMA scheme with no feedback at all. The random codebook and especially the correlated random codebook seems to represent the channel more appropriately, since its performance increases with the number of feedback bits. For a high number of feedback bits it outperforms the Fourier codebook and for a low number of feedback bits the performance is comparable to the one of the Fourier codebook.

\section{REFERENCES}

[1] G. Caire and S. Shamai (Shitz), "On the achievable throughput of a multiantenna Gaussian broadcast channel," IEEE Trans. Inf. Theory, vol. 49, no. 7, pp. 1691-1706, Jul. 2003.

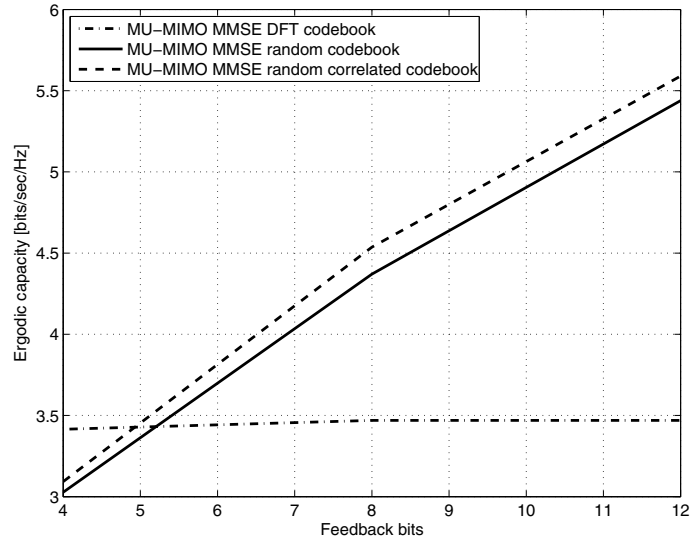

Fig. 5. Ergodic capacity of MU-MIMO with MMSE precoding and different codebooks vs. codebook size. The average SNR is fixed to $10 \mathrm{~dB}$ for each user.

[2] H. Weingarten, Y. Steinberg, and S. Shamai (Shitz), "The capacity region of the gaussian multiple-input multiple-output broadcast channel," IEEE Trans. Inf. Theory, vol. 52, no. 9, pp. 3936-3964, Sep. 2006.

[3] D. Gesbert, M. Kountouris, R. W. Heath, Jr., C. B. Chae, and T. Sälzer, "From single user to multiuser communications: Shifting the MIMO paradigm," IEEE Signal Process. Mag., vol. 24, no. 5, pp. 36-46, Sep. 2007.

[4] D. Gesbert, F. Tosato, C. van Rensburg, and F. Kaltenberger, UMTS Long Term Evolution: From Theory to Practice. Wiley and Sons, 2008, ch. Multiple Antenna techniques in LTE, in print.

[5] Philips, "Comparison between MU-MIMO codebook-based channel reporting techniques for LTE downlink," 3GPP TSG RAN WG1, Tech. Rep. R1-062483, Oct. 2006.

[6] - "System-level simulation results for channel vector quantisation feedback for MU-MIMO," 3GPP TSG RAN WG1, Tech. Rep. R1063028, Nov. 2006.

[7] C. B. Peel, B. M. Hochwald, and A. L. Swindlehurst, "A vector-perturbation technique for near-capacity multiantenna multiuser communication-part I: channel inversion and regularization," IEEE Trans. Commun., vol. 53, no. 1, pp. 195-202, Jan. 2005.

[8] R. de Lacerda, L. S. Cardoso, R. Knopp, M. Debbah, and D. Gesbert, "EMOS platform: real-time capacity estimation of MIMO channels in the UMTS-TDD band," in Proc. International Symposium on Wireless Communication Systems (IWCS), Trondheim, Norway, Oct. 2007.

[9] F. Kaltenberger, L. S. Cardoso, M. Kountouris, R. Knopp, and D. Gesbert, "Capacity of linear multi-user MIMO precoding schemes with measured channel data," in Proc. IEEE Intl. Workshop on Signal Processing Advances in Wireless Communications (SPAWC), Recife, Brazil, Jul. 2008.

[10] F. Kaltenberger, M. Kountouris, D. Gesbert, and R. Knopp, "Correlation and capacity of measured multi-user MIMO channels," in Proc. IEEE Intl. Symposium on Personal, Indoor and Mobile Radio Communications (PIMRC), Cannes, France, Sep. 2008.

[11] G. Bauch, J. Anderson, C. Guthy, M. Herdin, J. Nielsen, J. A. Nossek, P. Tejera, and W. Utschick, "Multiuser MIMO channel measurements and performance in a large office environment," in Proc. IEEE Wireless Comm. and Net. Conf., Hong Kong, Mar. 2007, pp. 1900-1905.

[12] G. W. K. Colman and T. J. Willink, "Limited feedback precoding in realistic MIMO channel conditions," in Proc. IEEE Int. Conf. on Comm. (ICC), Glasgow, Scotland, Jun. 2007, pp. 4363-4368.

[13] S. A. Jafar and S. Srinivasa, "On the optimality of beamforming with quantized feedback," IEEE Trans. Commun., vol. 55, no. 12, pp. $2288-2302,2007$.

[14] N. Jindal, "MIMO broadcast channels with finite-rate feedback," IEEE Trans. Inf. Theory, vol. 52, no. 11, pp. 5045-5060, 2006.

[15] R. de Francisco Martín, "Performance optimization of MIMO systems with partial channel state information," Ph.D. dissertation, Institut Eurecom, Sophia-Antipolis, France, 2008.

[16] A. Paulraj, D. Gore, and R. Nabar, Introduction to Space-Time Wireless Communications. Cambridge University Press, 2003. 